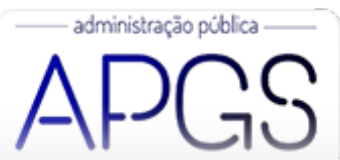

Administração Pública e Gestão Social ISSN: 2175-5787

apgs@ufv.br

Universidade Federal de Viçosa

Brasil

\title{
Avaliação da Eficiência dos Gastos Públicos com Educação dos Municípios de Santa Catarina
}

Rocha dos Santos, Rodolfo; Machado de Freitas, Marcelo; Flach, Leonardo

Avaliação da Eficiência dos Gastos Públicos com Educação dos Municípios de Santa Catarina

Administração Pública e Gestão Social, vol. 12, núm. 2, 2020

Universidade Federal de Viçosa, Brasil

Disponible en: http://www.redalyc.org/articulo.oa?id=351562414006

Esta obra está bajo una Licencia Creative Commons Atribución-NoComercial-SinDerivar 3.0 Internacional. 


\title{
Avaliação da Eficiência dos Gastos Públicos com Educação dos Municípios de Santa Catarina
}

\author{
The Efficiency of Public Spending on Education in The Municipalities of Santa Catarina \\ Evaluación de la Eficiencia de los Gastos Públicos con Educación de los Municipios de Santa Catarina \\ Rodolfo Rocha dos Santos \\ Universidade Federal Rural do Rio de Janeiro, Brasil \\ Redalyc: http://www.redalyc.org/articulo.oa? \\ rodolforsantos@ufrrj.br
}

Marcelo Machado de Freitas

Universidade Federal de Santa Catarina, Brasil

mmf.marcelofreitas@gmail.com

Leonardo Flach

Universidade Federal de Santa Catarina, Brasil

leonardo.flach@gmail.com

Recepción: 19 Diciembre 2017

Aprobación: 16 Octubre 2018

Publicación: 01 Abril 2020

\section{Resumo:}

Este artigo teve como objetivo verificar os fatores que afetam o nível de eficiência na aplicação dos recursos públicos em educação básica dos municípios do estado de Santa Catarina. Para tanto, utilizou-se o método não paramétrico de análise envoltória de dados. Posteriormente, aplicou-se um modelo de Regressão Tobit. Pode-se inferir, ao nível de significância de 10\%, que a qualidade de vida de um município afeta positivamente a eficiência na aplicação dos recursos públicos com educação dos municípios catarinenses. $\mathrm{O}$ produto interno bruto (PIB) apresentou uma relação negativa com a escala de eficiência, podendo-se afirmar que, ao nível de significância de 5\%, municípios que apresentaram maior poder econômico foram menos eficientes. Por fim, a densidade demográfica apresentou uma relação positiva com a eficiência dos municípios catarinenses ao nível de significância de $10 \%$, portanto, entende-se que municípios mais densamente povoados podem apresentar uma maior demanda em relação à educação, sem a necessidade de uma maior aplicação de recursos.

Palavras-Chave: Gastos públicos, Avaliação de desempenho, Educação.

\section{Abstract:}

This paper had the objective to verify the factors that affect the level of efficiency in the application of the public resources in basic education in the municipalities of the state of Santa Catarina, in Brazil. For this, the non-parametric method of data envelopement analysis was used. Subsequently, a Tobit regression model was applied. It can be inferred, at the level of significance of $10 \%$, that the quality of life of a municipality positively affects the efficiency in the application of public resources with education in the municipalities of Santa Catarina. The gross domestic product (GDP) presented a negative relation with the efficiency scale, and it can be affirmed that at the level of significance of $5 \%$, municipalities that presented greater economic power were less efficient. Finally, demographic density showed a positive relation with the efficiency of the municipalities of Santa Catarina at the level of significance of $10 \%$, it is understood that more densely populated municipalities can present a higher demand for education without the need of a greater application of resources.

KEYWORDs: Public Spending, Performance evaluation, Education.

\section{RESUMEN:}

Esta investigación tuvo como objetivo verificar los factores que afectan el nivel de eficiencia en la aplicación de los recursos públicos en educación básica de los municipios del Estado de Santa Catarina, Brasil. Para ello, se utilizó el método no paramétrico de análisis envoltorio de datos. Posteriormente, se aplicó un modelo de regresión Tobit. Se puede inferir, al nivel de significancia de $10 \%$, que la calidad de vida de un municipio afecta positivamente la eficiencia en la aplicación de los recursos públicos 
con educación de los municipios catarinenses. El producto interno bruto (PIB) presentó una relación negativa con la escala de eficiencia, pudiéndose afirmar que, al nivel de significancia de 5\%, municipios que presentaron mayor poder económico fueron menos eficientes. Por último, la densidad demográfica presentó una relación positiva con la eficiencia de los municipios catarinenses al nivel de significancia del 10\%, por eso, se entiende que municipios más densamente poblados pueden presentar una mayor demanda con educación sin la necesidad de una mayor aplicación de recursos.

Palabras Clave: Gastos públicos, Evaluación del desempeño, Educación.

\section{INTRODUÇão}

A educação é um direito de todos e tem fundamental importância para o crescimento econômico de uma nação. A existência de desigualdades no setor educacional, sobretudo nos componentes de oferta do serviço e na ineficiência na aplicação dos recursos públicos, reflete no desenvolvimento socioeconômico das regiões. Isto afeta a qualidade de vida da população (Silva et al., 2012) e também seu índice de desenvolvimento humano (IDH) (Dias, Cagnini, \& Camargo, 2014).

A manutenção do sistema educacional público exige uma grande quantidade de recursos, que muitas vezes não se encontram disponíveis. Neste sentido, é importante que estudos verifiquem se os entes públicos utilizam esses recursos de maneira eficiente. A avaliação da eficiência nos gastos públicos com educação auxilia no planejamento e na gestão. Um bom planejamento e gestão das políticas públicas possibilita melhorar a alocação dos recursos aplicados e torna o uso das despesas públicas mais racionais (Arretche, 1998).

Dentre as diversas metodologias que permitem avaliar a eficiência, Peña (2008) evidencia que o método de Análise Envoltória de Dados (Data Envelopment Analysis [DEA]) tem sido aplicado com sucesso em estudos de eficiência da administração pública. Esse método permite comparar os insumos (inputs) e os produtos (outputs) de cada unidade, e determina os índices de eficiência relativa de cada unidade analisada. De acordo com Curi et al. (2014), esses índices possibilitam delinear as melhores práticas, as unidades ineficientes e as mudanças necessárias nos níveis de insumos e produtos para que as unidades consideradas ineficientes tornem-se eficientes. Pode-se também identificar recursos ociosos ou inutilizados e auxiliar na formação de políticas de redução de custos.

O trabalho de Charnes, Cooper e Rhodes (1978) deu visibilidade ao DEA ao utilizar tal metodologia para mensurar a eficiência dos gastos de programas educacionais em escolas do estado americado do Texas. Desde então, inúmeros trabalhos voltados a esta temática surgiram no cenário acadêmico (Abbott \& Doucoliagos, 2003; Hauner, 2008; Faria, Jannuzzi, \& Silva, 2008; Lopes \& Toyoshima, 2008; Silva \& Almeida, 2012; Dias et al., 2014; Nuintin et al., 2014)

Estudos nacionais já avaliaram a eficiência dos gastos com educação de vários municípios brasileiros por meio da Análise Envoltória de Dados, como, por exemplo, o trabalho de Faria et al. (2008), que avaliou a eficiência dos gastos públicos com educação, cultura, saúde e habitação nos municípios fluminenses.

Apesar de o estado de Santa Catarina possuir o terceiro melhor IDH do país $(0,774)$ e a menor desigualdade de renda (Gini de 0,49), segundo dados do Atlas Brasil (2010), é importante aplicar um estudo voltado aos seus municípios para entender a sua eficiência em termos de aplicação dos recursos públicos com educação. Mesmo que o estado de Santa Catarina possua bons indicadores sociais e de desenvolvimento, isso não necessariamente significa que seus municípios estejam gastando os recursos de maneira eficiente. Compreender a aplicação dos recursos públicos com educação nos municípios de Santa Catarina pode auxiliar as futuras gestões de políticas públicas.

Tendo em vista a importância da destinação eficiente dos recursos aplicados na educação e a necessidade de se entender a eficiência da aplicação de tais recursos nos municípios catarinenses, o presente trabalho tem por objetivo verificar quais fatores afetam a eficiência da aplicação dos recursos públicos com educação nos municípios de Santa Catarina. Nesta direção, espera-se encontrar um ranking de eficiência dos municípios com os gastos com educação e verificar fatores externos que afetam a eficiência. 


\section{Referencial Teórico}

A educação pública costuma enfrentar diversos entraves em seu desenvolvimento. Um dos problemas comumente debatidos pela academia consiste na falta de eficiência na utilização dos recursos disponíveis. Lopes e Toyoshima (2008) explicam que uma maior alocação dos recursos públicos em educação, saneamento básico, saúde e habitação é fundamental para a melhoria do desenvolvimento e redução das disparidades existentes.

Altos níveis de educação melhoram o nível de capital humano de um país ao gerar crescimento econômico no longo prazo (Whiteley, 2000). São os gastos com educação que mais geram crescimento, ou melhor, é o investimento público com educação que eleva o nível de capital humano que, consequentemente, promove o crescimento da economia (Zoghbi et al., 2009).

Porém, somente conhecer a aplicação dos recursos públicos com provisões de bens e serviços não é suficiente. É necessário saber o resultado gerado por essa aplicação e se esse resultado poderia ser alcançado com menores custos para o contribuinte (Rezende, Cunha, \& Bevilacqua, 2010). Nem sempre o maior dispêndio de recursos resulta em uma maior qualidade de suas aplicações e é justamente nesse sentido a demanda por estudos que avaliem os resultados da aplicação dos recursos públicos (Will, 2014). Esses resultados, para Will (2014), podem ser medidos pela eficiência da aplicação dos recursos pelos agentes públicos.

O que seria eficiência? Penã (2008) explica que eficiência é a combinação ótima dos insumos e métodos necessários (inputs) no processo produtivo de maneira que resulte no máximo de produtos possíveis (outputs). Isto é, eficiência é a capacidade de fazer corretamente as ações, de minimizar a relação insumo e produto e, desse modo, otimizar a utilização de recursos. Mello et al. (2005) afirmam que eficiência consiste em comparar o que foi produzido, dados os recursos disponíveis, com o que poderia ter sido produzido com os mesmos recursos.

A mensuração da eficiência dos gastos em educação continua a ser tema de interesse da comunidade acadêmica. Estudos internacionais e nacionais buscaram conhecer a eficiência dos gastos públicos com educação. Inúmeras metodologias foram aplicadas para medir a eficiência, dentre elas a DEA. Charnes $e t$ al. (1978) desenvolveram a DEA a partir do trabalho de Farrel (1957) e, desde então, inúmeros trabalhos utilizaram-se dessa ferramenta de maneira satisfatória para medir a eficiência pública (Peña, 2008).

O próprio trabalho de Charnes et al. (1978) teve como objetivo o desenvolvimento de uma técnica para comparar a eficiência de escolas públicas, levando em consideração uma série de inputs utilizados e uma série de outputs gerados por uma unidade tomadora de decisão (uma escola). Na apresenta-se a síntese de alguns estudos internacionais sobre o tema. 


\begin{tabular}{|c|c|}
\hline Autor, ano e revista & Objetivos, Inputs, Outputs e Metodologia \\
\hline $\begin{array}{l}\text { (Gupta \& Verhoeven, } \\
\text { 2001) Journal of Policy } \\
\text { Modeling }\end{array}$ & $\begin{array}{l}\text { Utilizaram uma metodologia similar ao DEA (Free Disposal } \\
\text { Hull [FDH]) para mensurar a eficiência da educação em } 37 \\
\text { países africanos. Input para a educação: Os gastos per } \\
\text { capita com educação, expressos em poder de compra. } \\
\text { Outputs: A quantidade de matrículas em escolas primárias, } \\
\text { quantidade de matrículas no ensino fundamental e a taxa de } \\
\text { analfabetismo entre os adultos. Regressaram pelo Métodos } \\
\text { dos Minimos Quadrados e os resultados obtidos de eficiência } \\
\text { com alguns indicadores econômicos e financeiros permitiram } \\
\text { encontrar quais motivos levaram aos niveis de eficiência } \\
\text { encontrados. }\end{array}$ \\
\hline $\begin{array}{l}\text { (Abbott \& } \\
\text { Doucouliagos, 2003) } \\
\text { Economics of Education } \\
\text { Review }\end{array}$ & $\begin{array}{l}\text { Aplicaram o DEA para medir a eficiência do ensino e da } \\
\text { pesquisa em } 36 \text { universidades da Austrália. Diversos inputs } \\
\text { e outputs foram utilizados. Inputs: Quantidade de } \\
\text { funcionários acadêmicos (professores, assistentes, etc.) e } \\
\text { quantidade de funcionários administrativos. Ouputs para } \\
\text { ensino: Número de alunos equivalentes em tempo integral e } \\
\text { número de matriculas de pós-graduação e de graduação. } \\
\text { Ouputs para pesquisa: Gastos com pesquisa acadêmica. }\end{array}$ \\
\hline $\begin{array}{l}\text { (Hauner, 2008) World } \\
\text { Development }\end{array}$ & $\begin{array}{l}\text { Aplicou o DEA e a metodologia de Afonso, Schuknecth e } \\
\text { Tanzi (2005) para medir a eficiência dos gastos com } \\
\text { educação, saúde e seguridade social dos governos } \\
\text { sub-nacionais da Rússia. Input em educação: Gastos com } \\
\text { educação consolidados dos governos em cada região do país. } \\
\text { Outputs em educação: A cobertura da pré-escola, a } \\
\text { cobertura geral, primária, profissional (técnico) e profissional } \\
\text { secundário. }\end{array}$ \\
\hline $\begin{array}{l}\text { (Agasisti, 2011) } \\
\text { Education Economics }\end{array}$ & $\begin{array}{l}\text { Utilizou o DEA para medir a eficiência do ensino superior em } \\
\text { países europeus. Input: Gastos com educação em } \\
\text { percentual do PIB, ingressos anuais de alunos e número de } \\
\text { professores por estudante. Diversos outputs foram } \\
\text { utilizados, dentre eles a porcentagem da população entre } 25 \\
\text { e } 34 \text { anos com ensino superior e a quantidade de matrículas } \\
\text { de graduação. }\end{array}$ \\
\hline $\begin{array}{l}\text { (Sibiano \& Agasisti, } \\
\text { 2013) Journal of Public } \\
\text { Affairs }\end{array}$ & $\begin{array}{l}\text { Utilizaram o DEA para medir o grau de eficiência do ensino } \\
\text { fundamental em diferentes regiôes da Itália. Input: Número } \\
\text { de professores por estudante. Outputs: Scores de } \\
\text { Matemática obtidos no exame do Programa Internacional de } \\
\text { Avaliação de Estudantes (PISA) de } 2009 \text {. Numa segunda } \\
\text { análise, utilizaram a Regressão Tobit para conhecer os } \\
\text { fatores que estavam afetando o nivel de eficiência dessas } \\
\text { regiões. }\end{array}$ \\
\hline
\end{tabular}

Fonte: elaborado pelos autores (2015).

$\mathrm{Na}$ Figura 2 elaborou-se uma síntese de alguns estudos nacionais que abordaram a eficiência da educação pública em municípios brasileiros. 
Figura 2 - Relação de estudos nacionais que abordaram a eficiência dos gastos com educação

\section{Autor, ano, revista ou anais}

(Faria et al, 2008) Revista de Administração Pública (RAP)

(Lopes \& Toyoshima, 2008) Anais de Seminário sobre a economia mineira

\section{Objetivos, Inputs, Outputs e Metodologia}

Analisaram a relação entre as despesas sociais realizadas nos municípios do estado do Rio de Janeiro (gastos públicos com educação, cultura, saúde e saneamento) e indicadores da condição de vida da população residente nos mesmos, utilizando o método DEA. O trabalho criou uma série de modelos até selecionar dois modelos com maior poder de discriminação. Inputs: Gastos per capita com educação e cultura e rendimento médio mensal dos responsáveis pelos domicílios particulares permanentes. Outputs Taxa de alfabetização de 10 a 14 anos e proporção de crianças de 2 a 5 anos matriculadas em creches ou escolas de educação infantil.

Calcularam a eficiência técnica da gestão dos recursos com saúde e educação no estado de Minas Gerais utilizando a metodologia DEA. Verificaram os impactos e determinantes dessa eficiência. OS autores constataram que a eficiência da aplicação dos recursos públicos tende a elevar o bem-estar e reduzir a desigualdade do estado. Inputs: Gastos per capita com educação e cultura; renda média mensal dos chefes de familias. Outputs: Percentual de docentes com curso superior no ensino fundamental; taxa de alfabetização.

Aplicarama DEA para medir a eficiência dos gastos públicos com educação fundamental dos municípios do estado de Alagoas no período de 2007 a 2011 . Os autores evidenciaram nos resultados de sua pesquisa que os municipios que compuseram a fronteira de Wilbert \& D'Abreu, eficiência foram aqueles com as piores condições em termos de 2011) Advances in riqueza média e nivel educacional. Já os municípios menos Scientific and Applied

Accounting (ASAA) apresentaram os piores desempenhos no Índice de eficientes foram aqueles com a melhor condição em termos de PIB per capita e que apresentaram elevados gastos por aluno. Porém, Desenvolvimento da Educação Básica (IDEB) de 2011. Input: Gastos públicos com educação. Output: Nota dos municípios alagoanos no IDEB de 2011 para a $4^{a}$ e $8^{a}$ série do ensino fundamental em escolas municipais. Avaliaram a eficiência técnica dos gastos públicos municipais do Ceará em educação, saúde e assistência social, utilizando o método (Machado, Irffi, \& Benegas, 2011) Planejamento e Políticas Públicas

(Silva et al., 2012) Contabilidade, Gestão e Governança

(Silva \& Almeida, 2012) Planejamento e Políticas Públicas

(Dias et al., 2014) Anais do Congresso Brasileiro de Custos (ABC)
DEA. A eficiência relativa, pelo conjunto de todas as áreas, representou um bom desempenho dos municipios cearenses. Entretanto, ao analisar a eficiência por área, os municipios apresentam uma baixa eficiência técnica. Input: Gasto per capita com educação e cultura. Outputs: Número de estabelecimentos de educação infantil, taxa de alfabetização de educação infantil e taxa de escolarização.

Analisaram a eficiência de alocação dos recursos destinados a educação, saúde e habitação nos municípios do estado de Minas Gerais com o uso do método DEA. Os municípios mineiros apresentaram uma baixa média dos escores de eficiência. Inputs: Gasto per capita com educação e cultura e PIB per capita. Outputs: Taxa de atendimento das crianças de 4 a 6 anos, taxa de crescimento das crianças de 7 a 14 anos e taxa de atendimento de adolescentes de 15 a 17 anos.

Mensuraram a eficiência dos municípios do estado do Rio Grande do Norte no uso do Fundo de Manutenção e Desenvolvimento do Ensino Fundamental (Fundef) e relacionaram esta eficiência com os indicadores da avaliação educacional de 2005. Os autores utilizaram os métodos DEA e FDH para mensurar duas fronteiras de produção na educação, constatando uma baixa eficiência do gasto público na educação municipal. Uma redução do nivel de reprovação poderia ser alcançada com o aumento da eficiência do gasto na educação. Inputs: Recur'sos do Fundef destinados para educação municipal. Outputs: Número de alunos matriculados, número de professores, número de escolas e salas de aula. Identificaram a eficiência com que os administradores públicos investem em educação fundamental nos municípios do sudoeste do Paraná nos anos de 2009 e 2011, utilizando o método DEA. Os autores perceberam que do ano de 2009 para 2011 o número de municípios na fronteira de eficiência diminuiu, de $16,66 \%$ (em 2009) para 7\% (em 2011), mesmo com um aumento dos gastos. Porém, $31 \%$ dos municipios aumentaram seus scores de eficiência de um ano para o outro. Input: Despesas com educação fundamental. Outputs: Número de alunos, número de professores e escolas de cada município. 
Fonte: elaborado pelos autores (2015).

Assim como Penã (2008) evidencia, a DEA vem sendo utilizada satisfatoriamente para medir a eficiência da aplicação dos recursos públicos, inclusive os recursos voltados para a educação, tanto no cenário nacional quanto no cenário internacional.

\section{Procedimentos Metodológicos}

A metodologia da pesquisa pode ser dividida em duas etapas distintas. Na primeira, utiliza-se a DEA para construir os índices de eficiência dos gastos públicos com educação nos municípios do estado de Santa Catarina. Em seguida, realiza-se um modelo de Regressão Tobit.

\subsection{Análise Envoltória de Dados}

Conforme Lins e Meza (2000), o método DEA foi proposto por Charnes et al. (1978) a partir do trabalho de Farrell (1957). O método foi proposto para avaliar a eficiência inerente de Unidades Tomadoras de Decisões homogêneas (Decision Making Units [DMUs]), tendo como referencial a transformação de inputs em outputs (Charnes et al., 1978).

O método DEA fornece um resultado de eficiência relativa dentro do intervalo entre 0 e 1 para cada uma das DMUs. O método aponta aquelas que apresentam score igual a 1 , que serão consideradas benchmarkings. Aquelas que apresentam uma eficiência inferior são relativamente ineficientes. A fronteira de eficiência é composta pelas DMUs eficientes e é a partir dela que se define as metas de desempenho para as DMUs ineficientes (Macedo, Casa Nova, \& Almeida, 2009). As pontuações para unidades ineficientes pertencem ao conjunto de $[0,1[$, em que uma pontuação 0,$1 ; 0,2 ; 0,3 ;[\ldots]$ implica que o mesmo resultado poderia ser teoricamente produzido com apenas 10\%, 20\%,30\%, [...], respectivamente, dos recursos despendidos (Hauner, 2008).

A vantagem da utilização da DEA é por permitir avaliar o desempenho de organizações que operam em mercados onde os preços não estão disponíveis (Zhu, 2000). Nesses casos, os indicadores de desempenho habituais, como a rentabilidade e as taxas de retorno, não podem ser usados para medir o desempenho econômico de uma instituição com precisão (Abbott \& Doucoliagos, 2003). Will (2014) destaca que a sua utilização se adequa na utilização de insumos ou geração de produtos na qual seus valores não podem ser mensurados em termos monetários. Por conta disso, a DEA tem sido aplicada com sucesso em estudos sobre eficiência na Administração Pública (Penã, 2008).

Existem duas abordagens clássicas do modelo DEA: O Charnes, Cooper e Rhodes (CCR), também chamado de Constant Returns to Scale (CRS), que aceita como hipótese um retorno constante de escala (Charnes et al., 1978); e o modelo Banker, Charnes e Cooper (BCC), conhecido como Variable Returns to Scale (VRS), que considera situações de eficiência da produção com variação de escala, além de não assumir proporcionalidade entre inputs e outputs (Banker, Charnes, \& Cooper, 1984).

No modelo CCR há a suposição de axioma da proporcionalidade, ou seja, de que qualquer variação nos inputs acarreta em uma variação proporcional dos outputs (Tshaffon \& Meza, 2014). O modelo CCR visa maximizar a razão entre a combinação linear dos outputs e a combinação linear dos inputs, onde essa razão não apresente valores maiores que 1 para cada DMU (Mello et al., 2008).

Já o modelo BCC pressupõe que as DMUs assumam retornos variáveis de escala. Isso forma uma fronteira convexa, onde não há proporcionalidade entre inputs e outputs (Mello et al., 2008; Tshaffon \& Meza, 2014). Esse modelo permite a projeção de cada DMU ineficiente sobre a fronteira de eficiência (envoltória) estabelecida conforme os diferentes portes de DMUs. Deste modo, a produtividade máxima é estabelecida conforme as escalas de produção. O BCC surgiu na divisão da eficiência do modelo CCR em duas 
componentes: A eficiência técnica e a eficiência de escala (Belloni, 2000; Macedo, Casa Nova, \& Almeida, 2009; Macedo, Cruz, \& Ferreira, 2011; Will, 2014).

Além das abordagens clássicas mencionadas anteriormente, o modelo DEA admite orientações para inputs e para outputs. $\mathrm{Na}$ orientação input verifica-se o quanto conseguiria reduzir o número de recursos para se produzir o mesmo nível de produtos. Já na orientação output verifica-se o quanto se pode maximizar o nível de produção se for mantido o mesmo nível de insumos (Tshaffon \& Meza, 2014).

A escolha das variáveis para o cálculo da eficiência relativa ocorreu com base na função alocativa do Estado, orientando-se nas necessidades de atendimento aos cidadãos no âmbito da educação. Além disso, ocorreu uma na análise de diferentes referenciais teóricos sobre o assunto. Tomou-se como referência alguns quesitos já validados por Lopes e Toyoshima (2008), Faria et al. (2008), Machado, Irffi e Benegas (2011), Silva et al. (2012), Silva e Almeida (2012) e Wilbert e D’abreu (2013).

As variáveis, divididas em inputs e outputs, são apresentadas no Figura 3. Como input selecionou-se o gasto per capita com educação e cultura $(\mathrm{GpcEdu \& C})$ dos municípios e o rendimento médio mensal dos responsáveis pelos domicílios particulares permanentes (Renda) de cada município. Vale ressaltar que a variável $\mathrm{GpcEdu} \& \mathrm{C}$ só levou em consideração os gastos das contas municipais com Ensino Fundamental, Educação Infantil e outras despesas na Função Educação. Selecionou-se tal variável para atender o objetivo do trabalho. A variável Renda foi utilizada como in put para ponderar o impacto da renda pessoal sobre a procura por serviços de educação (Faria et al., 2008; Lopes \& Toyoshiba, 2008). Tal variável deve ser considerada uma variável exógena, ambiental ou não discricionária (Lins \& Meza, 2000).

\begin{tabular}{|l|l|}
\hline \multicolumn{1}{|c|}{ Inputs } & \multicolumn{1}{c|}{ Autores que as utilizaram } \\
\hline \hline GpcEdu\&C & $\begin{array}{l}\text { Faria } \text { et al. (2008); Lopes e Toyoshima, (2008); Machado } \text { et al. (2011); Silva } \text { et al. } \\
\text { (2012) e Wilbert e D'abreu (2013). }\end{array}$ \\
\hline \hline Renda & Faria et al. (2008); Lopes e Toyoshima (2008). \\
\hline \hline \multicolumn{1}{|c|}{ Outputs } & \multicolumn{1}{c|}{ Autores que as utilizaram } \\
\hline \hline TaxAt0-5\% & Faria et al. (2008); Silva et al. (2012) \\
\hline \hline TaxAt5-6\% & Silva et al. (2012) \\
\hline \hline TaxAt7-14\% & Silva et al. (2012) \\
\hline \hline TaxAlf11-14 anos \% & Faria et al. (2008); Machado et al. (2011) \\
\hline
\end{tabular}

Figura 3 - Variáveis da pesquisa

Fonte: elaborado pelos autores (2015).

Como variáveis "candidatas" para a output selecionou-se a taxa de atendimento às crianças de 0 a 5 anos (TaxAt0-5\%) de cada município, a taxa de atendimento às crianças de 5 a 6 anos (TaxAt5-6\%), a taxa de atendimento às crianças de 7 a 14 anos (TaxAt7-14\%) e taxa de alfabetização de crianças de 11 a 14 anos (TaxAlf1 1-14\%).

A escolha das variáveis TaxAt0-5\%, TaxAt5-6\% e TaxAt7-14\% ocorreu devido à responsabilidade delegada aos municípios pela Constituição Brasileira de $1988 \mathrm{em}$ seu art. 211, $\S 2^{\circ}$, quanto à educação fundamental e infantil.

A escolha da taxa de alfabetização de crianças de 11 a 14 anos como variável de output ocorreu em razão da mesma estar diretamente ligada ao fato de que a educação voltada para tal faixa etária é de responsabilidade dos municípios, por mais que ainda exista uma participação de verbas estaduais (Faria et al., 2008).

Optou-se por utilizar a orientação output, que mede o quanto a produção pode ser expandida sem que sejam alterados os inputs (no caso, o quanto as taxas de atendimento e alfabetização ainda podem ser ampliadas para os municípios ineficientes). Optou-se, também, pela aplicação do modelo de Retornos Variáveis de Escala (VRS), de Banker et al. (1984), de acordo com o que é recomendado por Souza e Gasparini (2006), com o intuito de considerar a disparidade de tamanho existente entre os municípios catarinenses.

Lins e Moreira (2000) levantaram alguns aspectos que devem ser respeitados na seleção de variáveis a serem introduzidas no modelo DEA: Observar se a variável abarca informação necessária que já não tenha sido compreendida em outras variáveis, verificar se a variável está relacionada ou contribui com ao menos um dos 
objetivos da aplicação, observar se as variáveis possuem dados confiáveis e seguros, e se a variável explica a eficiência de uma DMU. Os autores ainda complementam que outro critério para seleção de variável é o impacto dela na discriminação das DMUs. Tendo em vista a finalidade de selecionar as variáveis que deverão ser excluídas do modelo, é necessário testar o poder de discriminação das mesmas. A presente pesquisa guiouse por tais orientações.

O cálculo dos scores de eficiência foram feitos com o software Open Sourcer DEA (OSDEA) versão 0.2. Após calcular a escala de eficiência dos municípios de Santa Catarina, realizou-se um teste de correlação entre essa escala e as variáveis de inputs $\mathrm{Gpc} \& \mathrm{C}$ e Renda para entender a relação dessas variáveis com a eficiência dos municípios.

Posteriormente, os indicadores de eficiência foram utilizados como variáveis dependentes em um modelo de Regressão Tobit, com o intuito de avaliar quais variáveis constroem uma relação de significância com tais indicadores. Segundo Hoff (2007), tanto o modelo de Regressão Tobit quanto o modelo de Mínimos Quadrados Ordinários (MQO) são suficientes para o chamado "segundo estágio" da Análise Envoltória de Dados. Porém, Sueyoshi, Goto e Omi (2010) afirmam que o modelo de Regressão Tobit é mais adequado para a segunda fase da DEA pois o MQO acaba tornando-se tendencioso pela característica truncada da eficiência. Assim, nesta pesquisa optou-se por utilizar o modelo de Regressão Tobit. O modelo da regressão é apresentado na equação a seguir, assim como as variáveis independentes.

Onde: é a constante da função, são os coeficientes estimados da regressão, é o fator de erro da regressão e $\mathrm{X}_{1} ; \mathrm{X}_{2}$ e $\mathrm{X}_{3}$ são as variáveis independentes do modelo definidas a seguir: $\mathrm{X}_{1}$ - Índice de Desenvolvimento Humano Municipal (IDHM); $\mathrm{X}_{2}$ - Produto Interno Bruto do Município (PIB); $\mathrm{X}_{3}$ - Densidade demográfica.

A variável IDHM foi utilizada como proxy para o bem-estar social. Ou seja, espera-se que quanto maior o IDHM apresentado pelo município, maior seja o índice de eficiência alcançado pelo mesmo. Isto significa que se espera que o bem-estar social influencie na melhor aplicação dos recursos por parte dos administradores públicos, cuja variável também foi utilizada no trabalho de Lopes e Toyoshima (2008).

Os conselhos municipais em educação têm como função estabelecer metas e diretrizes para a política pública educacional do município. Espera-se que a participação desses conselhos reflita em um maior controle e fiscalização dos recursos aplicados, o que resulta em efeitos positivos sobre a eficiência no gasto com educação (Silva \& Almeida, 2012). Silva e Almeida (2012) utilizaram essa variável na análise da segunda fase da DEA em sua pesquisa.

O PIB dos municípios catarinenses foi utilizado como proxy para entender se as características econômicas influenciam na escala de eficiência. Espera-se que o crescimento do PIB municipal permita uma maior dedicação dos prefeitos na aplicação dos gastos com educação. Ou seja, com uma maior base de arrecadação municipal as prefeituras se dedicariam mais na aplicação dos recursos, e tal fato geraria efeitos positivos na eficiência dos gastos públicos com educação (Silva \& Almeida, 2012). Silva e Almeida (2012) também fizeram uso dessa variável para a análise da chamada segunda fase da DEA.

Por fim, espera-se que exista uma relação negativa da escala de eficiência com a densidade demográfica. Parte-se do pressuposto que o aumento populacional dos municípios signifique o aumento da demanda na educação. Isso faz com que municípios que apresentem uma maior densidade populacional possam ter dificuldades na gestão dos recursos públicos, inclusive os destinados à educação (Silva \& Almeida, 2012). Tanto Silva e Almeida (2012) quanto Lopes e Toyoshima (2008) utilizaram essa variável na análise de fatores explicativos para a escala de eficiência.

\subsection{Universo, amostra e base de dados}

A amostra do trabalho foi composta por 277 municípios do total de 295 do estado de Santa Catarina. Não foi utilizada toda a população de municípios pelo fato de não ter sido encontrado um ou mais dados para 
os municípios de Arroio Trinta, Balneário Piçarras, Balneário Rincão, Bandeirante, Bom Jesus do Oeste, Herval d'Oeste, Iomerê, Ipira, Lajeado Grande, Ouro, Peritiba, Pescaria Brava, Presidente Castello Branco, São Bernardino, São Miguel da Boa Vista, Serra Alta, Tigrinhos e Treviso. Porém, a amostra compreende 93,90\% de todos os municípios do estado.

As variáveis socioeconômicas e estatísticas da educação foram obtidas no Atlas do Desenvolvimento Humano, que utiliza dados secundários do Instituto Brasileiro de Geografia (IBGE), Instituto de Pesquisa Econômica Aplicada (IPEA), Instituto Nacional de Estudos e Pesquisas Educacionais Anísio Teixeira (INEP). A variável PIB foi obtida diretamente da base de dados do IBGE e os dados referentes aos gastos públicos com educação de cada município foram obtidos junto a Secretaria do Tesouro Nacional (STN). Os dados correspondem ao ano de 2010, quando foi realizado o último censo, por conta da falta de disponibilidade de informações mais atuais dos municípios catarinenses para todas as variáveis selecionadas.

\section{ANÁlise dos Resultados}

De acordo com o procedimento metodológico sugerido por Lins e Meza (2000), diversos modelos foram gerados por meio da combinação das variáveis de outputs, com o objetivo de conhecer o modelo que mais discrimine as DMUs. Vale ressaltar que a construção dos modelos foi pautada nos outputs por conta da orientação adotada pelo trabalho, que visa maximizá-los mantendo os inputs constantes. As combinações das variáveis permitiram construir 15 modelos distintos. $\mathrm{Na}$ Tabela 1 , a seguir, descreve-se os cinco modelos que apresentaram a maior discriminação entre as DMUs, mensurada por meio da diferença interquartil e do desvio padrão.

Tabela 1 - Modelos mais discricionários entre os 15 modelos elaborados

\begin{tabular}{|c|c|c|c|c|c|}
\hline Modelos & Média & DP & Mín. & Máx & Interv.IQ \\
\hline Mod L-GpcEdu\&C-Renda-TaxAt0-5 \% & 0,710 & 0,147 & 0,315 & 1,00 & $20,37 \%$ \\
\hline Mod M-GpcEdu\&C-Renda-TaxAt5-6 \% & 0,937 & 0,063 & 0,544 & 1,00 & $7,84 \%$ \\
\hline Mod F-GpcEdu\&C-Renda-TaxAt0-5\%-TaxAt5-6\% & 0,940 & 0,062 & 0,544 & 1,00 & $7,67 \%$ \\
\hline Mod B-GpcEdu\&C-Renda-TaxAt0-5\%-TaxAt5-6\%-TaxAt7-14\% & 0,986 & 0,013 & 0,929 & 1,00 & $2,16 \%$ \\
\hline Mod K-GpcEdu\&C-Renda-TaxAt5-6 \%-TaxAt7-14 \% & 0,985 & 0,013 & 0,929 & 1,00 & $2,02 \%$ \\
\hline
\end{tabular}

Fonte: elaborado pelos autores (2015).

Assim, optou-se pelo Modelo L para a construção da escala de eficiência dos municípios de Santa Catarina. Ele traz como output a taxa de atendimento às crianças de 0 a 5 anos. Vale ressaltar que Faria et al. (2008) aplicaram essa metodologia e chegaram ao mesmo modelo com maior poder de discriminação das DMUs.

A Tabela 2 apresentada a seguir traz as estatísticas descritivas da eficiência atingida pelos municípios de Santa Catarina pela perspectiva por mesorregião. A mesorregião do Vale do Itajaí foi a que apresentou a maior eficiência média dentro das seis mesorregiões. Pode-se destacar que, com exceção da Grande Florianópolis, todas as mesorregiões apresentaram municípios eficientes. E por fim, o município que apresentou a pior escala de eficiência foi Xavantina, localizado no Oeste Catarinense. 
Tabela 2 - Estatísticas descritivas da eficiência por mesorregião

Mesorregião
Serrana
Oeste Catarinense
Vale do Itajaí
Grande Florianópolis
Norte Catarinense
Sul Catarinense
Santa Catarina

Eficiência Modelo L

$\begin{array}{lllll}\text { Média } & \begin{array}{l}\text { Desv. } \\ \text { Padrão }\end{array} & \text { Mínimo } & \text { Máximo } & \begin{array}{l}\text { Menor } \\ \text { Eficiência }\end{array} \\ 0,7188 & 0,1457 & 0,4783 & 1,0000 & \text { São Joaquim } \\ 0,6956 & 0,1483 & 0,3159 & 1,0000 & \text { Xavantina } \\ 0,7580 & 0,1442 & 0,3294 & 1,0000 & \text { Botuverá } \\ 0,6534 & 0,1438 & 0,3579 & 0,9103 & \text { Alfredo Wagner } \\ 0,6922 & 0,1466 & 0,4777 & 1,0000 & \text { Timbó Grande } \\ 0,7159 & 0,1296 & 0,4437 & 1,0000 & \text { Passo de Torres } \\ 0,7097 & 0,1468 & 0,3159 & 1,0000 & \text { Xavantina }\end{array}$

Fonte: dados da pesquisa (2015).

Os municípios que compuseram a fronteira de eficiência e foram considerados benchmarks para os municípios ineficientes, foram: Salto Veloso, Major Vieira, Rio Rufino, São José do Cerrito, Braço do Trombudo, Gravatal, Massaranduba, Calmon, Imaruí, Saltinho, Monte Castelo. Dentre eles, o município de Saltinho ficou entre os cem menores GpcEdu\&C do estado de Santa Catarina. E os municípios de Major Vieira, Monte Castelo, Imaruí, Massaranduba, Gravatal, ficaram entre os duzentos municípios que tiveram os menores GpcEdu\&C.

Os municípios de Massaranduba e Gravatal apresentaram os menores GpcEdu\&C do estado de Santa Catarina. Eles atingiram altas taxas de atendimento a crianças de 0 a 5 anos para o nível atual de investimento. Tal fato levou-os para a eficiência, diferentemente de Monte Castelo, que apresentou TaxAt0-5\% baixa. Porém, foi considerada eficiente pelo seu nível de GpcEdu\&C. Os municípios de Piratuba, Bocaina do Sul, Zortéa, Capão Alto e Macieira são os cinco que tiveram os maiores GpcEdu\&C e se apresentaram como ineficientes. Esses resultados demonstram que a eficiência não está relacionada a maior ou menor disponibilidade de recursos. Municípios podem gastar muito e, simultaneamente, gastar de forma equivocada os seus recursos. Outros podem gastar pouco, mas de forma eficiente, ou seja, aplicando bem os seus recursos.

Aplicando o Teste de Correlação de Pearson entre o índice de eficiência calculado pelo Modelo L e a variável GpcEdu\&C, atinge-se um valor de $\mathrm{r}=0,0104$, muito próximo de zero. Realizou-se um teste $\mathrm{t}$ para o coeficiente de correlação, assumindo-se como H0: $r=0$ e H1: $r \neq 0$. Obteve-se um valor de $t=0,1733$, ou um p-valor $=0,8626$, não podendo rejeitar a hipótese nula, indicando que o índice de eficiência calculado pelo Modelo L e a variável GpcEdu\&C não são correlacionados. A Figura 1 demonstra esse resultado por meio da dispersão dos GpcEdu\&C e a eficiência atingida por cada município, facilitando a visualização da inexistência da correlação entre as variáveis. Esse resultado corrobora com os estudos de Faria et al. (2008), Will (2014) e Dias et al. (2014), que apontaram que não existe uma relação entre os gastos com educação e os índices de eficiência.

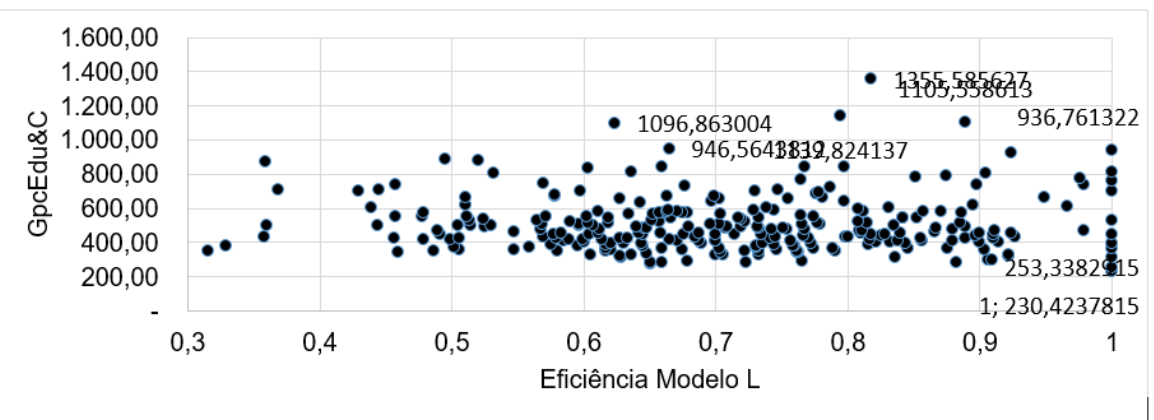

Figura 4 - GpcEdu\&C x Modelo L

Fonte: elaborado pelos autores (2015). 
Utilizou-se a variável Renda no modelo com o intuito de ponderar o impacto da renda pessoal na procura por educação. Ou seja, espera-se que municípios que apresentem um rendimento médio mensal dos responsáveis pelos domicílios particulares permanentes maiores busquem por mais educação, pressionando uma melhor aplicação dos recursos com essa função. Para o modelo adotado neste trabalho, essa maior busca resultaria em uma maior taxa de atendimento às crianças de 0 a 5 anos. Os municípios de Calmon e São José do Cerrito apresentaram os menores valores com rendimento médio mensal dos responsáveis pelos domicílios particulares permanentes do estado de Santa Catarina, os quais foram considerados eficientes pelo Modelo L. Já os municípios que apresentaram os vinte maiores rendimentos médios mensais dos responsáveis pelos domicílios particulares permanentes foram ineficientes, de acordo com o modelo adotado.

O Teste de Correlação de Pearson entre o índice de eficiência calculado pelo Modelo L e a variável Renda chegou ao valor de $\mathrm{r}=0,1321$. Realizou-se um teste $\mathrm{t}$ para o coeficiente de correlação, assumindo como $\mathrm{H} 0$ : $\mathrm{r}=0$ e H1: $\mathrm{r} \neq 0$. Obteve-se um valor de $\mathrm{t}=2,2104$, e um $\mathrm{p}$-valor $=0,0279$, podendo rejeitar a hipótese nula ao nível de significância de 5\%. Ou seja, o índice de eficiência calculado pelo Modelo L e a variável Renda são correlacionados positivamente, por mais que seja uma correlação consideravelmente fraca. Tal resultado corrobora com os achados do trabalho de Lopes e Toyoshiba (2008).

No Figura 2 percebe-se a dispersão entre a variável Renda e a eficiência do Modelo L, com a correlação entre o índice de eficiência calculado pelo Modelo L e a variável Renda.

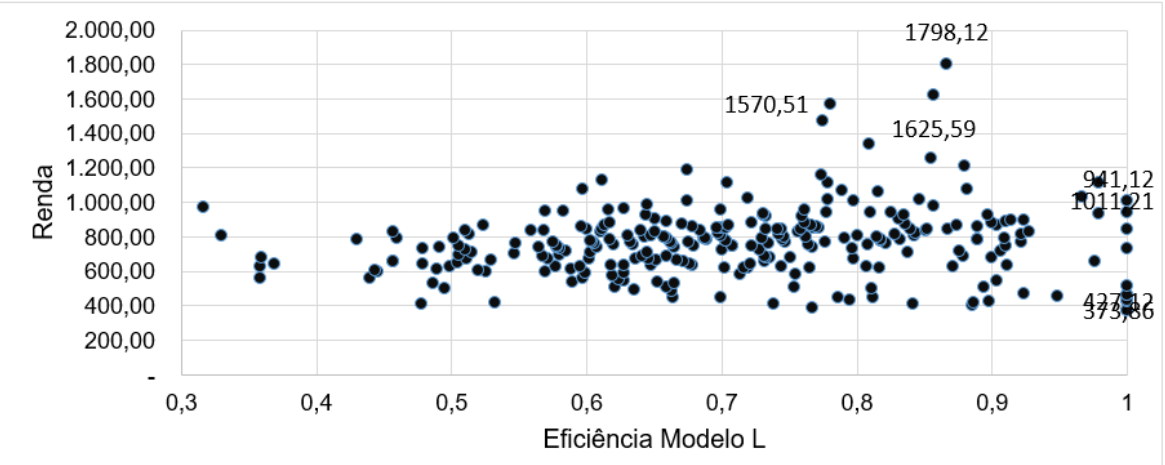

Figura 2 - Renda x Eficiência do Modelo L

Fonte: elaborado pelos autores.

\subsection{Modelo de Regressão Tobit}

Para a chamada "segunda fase" da DEA foi utilizado o Modelo de Regressão Tobit, no intuito de estabelecer quais das variáveis selecionadas são significantes para explicar a eficiência obtida pelo Modelo L. Utilizouse o software Gretl para rodar o modelo. Foram aplicados erros padrão robustos, por conta da presença de heterocedasticidade, característica do modelo de Regressão Tobit (Silva \& Almeida, 2012). A Tabela 3 traz os resultados obtidos pelo modelo de Regressão Tobit.

Tabela 1 - Modelos mais discricionários entre os 15 modelos elaborados

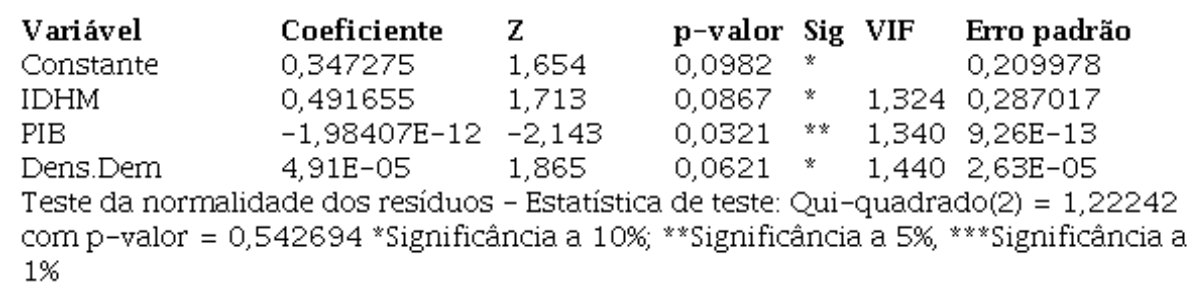


Fonte: elaborado pelos autores (2015).

O p-valor das estatísticas Z das variáveis do modelo de Regressão Tobit apresentou significância para as variáveis IDHM, PIB e densidade demográfica. O IDHM mostrou uma relação positiva com a escala de eficiência calculada pelo Modelo L, podendo afirmar que, ao nível de significância de $10 \%$, municípios que apresentam um melhor bem-estar social tendem a aplicar melhor seus recursos com educação. Esse resultado é explicado pelo fato de que municípios que apresentam melhores indicadores de qualidade de vida costumam manter como meta uma educação de qualidade. Para obter tais resultados, precisam ser eficientes na aplicação dos recursos disponíveis para esta finalidade. Os resultados deste estudo apoiam os resultados encontrados por Lopes e Toyoshima (2008).

Já o PIB apresenta uma relação negativa com a escala de eficiência calculada pelo Modelo L, resultado oposto ao estudo de Silva e Almeida (2012). Os autores encontraram que essa variável apresenta relação positiva com a eficiência. Para o modelo testado no presente trabalho, o aumento da renda dos munícipios, tendo o PIB como proxy, tende a gerar uma diminuição da eficiência da aplicação dos recursos públicos com educação. Tal resultado pode ser afirmado ao nível de significância de 5\%.

A variável Densidade Demográfica apresentou uma relação positiva com a escala de eficiência calculada pelo Modelo L. Pode-se inferir, ao nível de significância de 10\%, que municípios mais densamente povoados apresentam um índice de eficiência maior, resultado oposto ao esperado. Isso pode ocorrer em função de uma maior densidade populacional representar uma maior demanda por educação, sem que haja necessidade de uma maior alocação de recursos. No caso do Modelo L, isto representa uma maior procura por educação para crianças de 0 a 5 anos. No trabalho de Silva e Almeida (2012) essa relação é inversa. Os autores explicam que seus resultados podem ocorrer pelo fato de que municípios mais densamente povoados podem representar maiores gastos e dificuldades de atender o crescimento na demanda de bens e serviços públicos. Os resultados dessa pesquisa corroboram com os resultados de Lopes e Toyoshima (2008), que também encontraram relação positiva entre a escala de eficiência e a densidade demográfica.

Quanto ao modelo, por mais que o cálculo do IDH leve em consideração o PIB, nenhuma variável apresentou problemas de colinearidade de acordo com os Fatores de Inflacionamento da Variância (VIF). Em relação à normalidade do modelo, o teste qui-quadrado mostrou um p-valor de 0,542694 para uma hipótese nula de que os resíduos seguem uma distribuição normal, permitindo concluir que o modelo segue tal distribuição.

\section{CONSIDERAÇÕES FINAIS}

Saber o quanto se aplica para cada função governamental e o que é gerado por tal aplicação não é uma informação suficiente para entender os resultados alcançados pelos entes públicos. É necessário entender a eficiência de como essa aplicação de recursos é alcançada para a melhoria da gestão governamental.

Para tanto, o presente trabalho construiu uma escala de eficiência dos municípios do estado de Santa Catarina utilizando-se como ferramenta a Análise Envoltória de Dados e verificou quais os fatores externos que afetam essa escala de eficiência. Vários modelos foram construídos com as variáveis de inputs e outputs selecionadas, utilizando-se a DEA-VRS e orientação output. Dentre eles utilizou-se aquele com o maior poder de discriminação das DMUs. Posteriormente foi calculada a correlação entre a escala de eficiência com as variáveis GpcEdu\&C e Renda. Por fim, a escala de eficiência calculada foi regressada com as variáveis IDHM, presença de conselhos municiais de educação, PIB municipal, PIB municipal per capita e densidade demográfica utilizando um modelo de Regressão Tobit.

O coeficiente de correlação de Pearson não apresentou correlação entre a escala de eficiência construída e a variável GpcEdu\&C, mostrando que a eficiência da aplicação dos recursos públicos com educação independe da quantidade de recurso aplicada. Este trabalho corrobora com os resultados encontrados por Faria et al. 
(2008), Will (2014) e Dias et al. (2014). Os autores também mostram em seus resultados que a eficiência não está relacionada a um maior ou menor nível de gasto.

Por outro lado, o coeficiente de Pearson mostrou que houve correlação entre a escala de eficiência e a variável Renda. Mesmo que a correlação tenha sido fraca, isto mostra que há uma relação positiva entre o crescimento do rendimento médio mensal dos responsáveis pelos domicílios particulares permanentes e a eficiência dos gastos públicos com educação. Esse resultado corrobora com o estudo de Lopes e Toyoshima (2008).

Pode-se afirmar que, ao nível de significância de $10 \%$, um maior bem-estar social tende a gerar uma maior eficiência na aplicação dos recursos públicos com educação. Uma das justificativas plausíveis é que municípios que apresentam uma melhor qualidade de vida buscam por uma educação de qualidade, o que torna a aplicação dos recursos públicos com educação básica (de 0 a 5 anos) mais eficiente. Desta maneira, os resultados corroboram com Lopes e Toyoshima (2008).

A variável PIB apresentou uma relação negativa com a escala de eficiência construída. Infere-se, ao nível de significância de 5\%, que municípios com maior renda ou economia tendem a ser menos eficientes na aplicação dos seus recursos com educação.

Ao nível de significância de $10 \%$, pode-se perceber que a densidade demográfica dos municípios apresenta um efeito positivo nos seus índices de eficiência. Isso pode ser explicado pelo fato de que municípios mais densamente povoados tendem a ter uma maior demanda por educação sem que ocorra, obrigatoriamente, a necessidade de uma maior aplicação de recursos. Isso leva o município a tornar-se eficiente, conforme a metodologia desta pesquisa. Este resultado corrobora os estudos de Lopes e Toyoshima (2008), mas diverge do resultado de Silva e Almeida (2012).

Os resultados da presente pesquisa podem ser inferidos somente sobre os municípios de Santa Catarina e não devem ser generalizados para municípios de outros estados. Entretanto, considerações obtidas com a aplicação do modelo de Regressão Tobit concordam com trabalhos realizados em outros estados brasileiros. Isso pode indicar que algumas variáveis de fato são significativas para explicar a eficiência dos municípios. Sugere-se a utilização dessas variáveis em pesquisas futuras para saber se outros estados também apontam tais resultados.

Destacam-se como limitações deste trabalho as variáveis escolhidas para seus modelos, o modelo e orientação selecionados para construir a escala de eficiência dos municípios catarinenses, já que o DEA é sensível a essas características.

Por fim, espera-se que esse trabalho seja uma motivação para pesquisas futuras. Sugere-se a construção de modelos que utilizem outras variáveis, modelos DEA com orientação diferente e até mesmo um outro método estatístico para medir a eficiência da aplicação dos recursos públicos com educação, para o próprio estado de Santa Catarina e para outros estados brasileiros. Como última sugestão, sugere-se que os municípios elencados como eficientes sejam analisados qualitativamente, procurando conhecer quais práticas tais municípios adotam.

\section{REFERÊNCIAS}

Abbott, M., Doucouliagos, C. (2003). The efficiency of Australian universities: a data envelopment analysis. Economics of Education review, 22(1), 89-97.

Agasisti, T. (2011). Performances and spending efficiency in higher education: a European comparison through non\# parametric approaches. Education Economics, 19(2), 199-224.

Arretche, M. T. (1998). Tendências no estudo sobre avaliação. Avaliação de politicas sociais: uma questão em debate, 3, 29-49.

Banker, R. D., Charnes, A., Cooper, W. W. (1984). Some models for estimating technical and scale inefficiencies in data envelopment analysis. Management science, 30(9), 1078-1092. 
Belloni, J. Â. (2000). Uma metodologia de avaliação da eficiência produtiva de universidades federais brasileiras (Tese de Doutorado, Universidade Federal de Santa Catarina).

Atlas do desenvolvimento humano no Brasil (2010). Disponível em http://www.atlasbrasil.org.br

Charnes, A., Cooper, W. W., Rhodes, E. (1978). Measuring the efficiency of decision making units. European journal of operational research, 2(6), 429-444.

Curi, M. A. (2015). Eficiência de universidadesfederais no uso de recursos renováveis. (Tese de Doutorado, Universidade Federal de Lavras, Lavras, MG, Brasil).

Dias, H. L., Cagnini, W., Camargo, S. R. (2014, nov). Análise da eficiência dos gastos públicos com educação nos municípios do Sudoeste do Paraná. Artigo apresentado no XXII Congresso Brasileiro de Custos, Natal, RN. Artigo recuperado em http://anaiscbc.emnuvens.com.br/anais/article/view/3770/3771

Faria, F. P., Jannuzzi, P. D., Silva, S. D. (2008). Eficiência dos gastos municipais em saúde e educação: uma investigação através da análise envoltória no estado do Rio de Janeiro. Revista de administração pública, 42(1), 155-177.

Farrell, M. J. (1957). The measurement of productive efficiency. Journal of the Royal Statistical Society. Series A (General), 120(3), 253-290

Gupta, S.,Verhoeven, M. (2001). The efficiency of government expenditure: experiences from Africa. Journal of policy modeling, 23(4), 433-467

Hauner, D. (2008). Explaining differences in public sector efficiency: evidence from Russia's regions. World Development, 36(10), 1745-1765.

Hoff, A. (2007). Second stage DEA: Comparison of approaches for modelling the DEA score. European Journal of Operational Research, 181(1), 425-435.

Lins, M. P. E., Meza, L. A. (2000). Análise envoltória de dados: e perspectivas de integração no ambiente do Apoio à Decisão. COPPE/UFRJ.

Lins, M. P. E., Moreira, M. C. B. (2000). Implementação com Seleção de Variáveis em Modelos DEA. In: Lins, M. P. E., Meza, L. A. Análise envoltória de dados: e perspectivas de integração no ambiente do Apoio à Decisão (pp. 37-52). COPPE/UFRJ.

Lopes, L. S., Toyoshima, S. H. (2008). Eficiência técnica municipal na gestão dos gastos com saúde e educação em Minas Gerais: seus impactos e determinantes. Seminário sobre a economia mineira, 13, 1-24.

Macedo, M. A. D. S., Casa Nova, S. P. D. C., de Almeida, K. (2010). Mapeamento e análise bibliométrica da utilização da Análise Envoltória de Dados (DEA) em estudos em contabilidade e administração. Contabilidade, Gestão e Governança, 12(3)

Macedo, M. A. D. S., Cruz, C., Ferreira, A. C. S. (2011). Índice de Desenvolvimento Sustentável: uma análise apoiada em DEA para os municípios do Estado do Rio de Janeiro. Gestão \& Regionalidade, 27(81), 19-31.

Machado, S. P., Jr., Irffi, G. I., Benegas, M. B. B. (2011). Análise da eficiência técnica dos gastos com educação, saúde e assistência social dos municípios cearenses. Planejamento e Políticas Públicas, (36).

Mello, J. C. C. B. S., Meza, L. A., Gomes, E. G., Neto, L. B. (2005). Curso de análise de envoltória de dados. Simpósio Brasileiro de Pesquisa Operacional, 37, 2521-2547.

Mello, J. S., Angulo-Meza, L., Gomes, E. G., Fernandes, A. J. S., Neto, L. B. (2008). Estudo não paramétrico da relação entre consumo de energia, renda e temperatura. IEEE Latin America Transactions, 6, 153-161.

Nuintin, A. A. (2014). Eficiência da aplicação de recursos públicos nas universidades federais (Tese de Doutorado, Universidade Federal de Lavras).

Peña, C. R. (2008). Um Modelo de Avaliaçăo da Eficięncia da Administraçăo Pública através do Método Análise Envoltória de Dados (DEA). Revista de Administração Contemporânea, Curitiba, Paraná, 12(1), 83-106.

Rezende, F., Cunha, A., Bevilacqua, R. (2010). Informações de custos e qualidade do gasto público: lições da experiência internacional. Revista de Administração Pública, 44(4), 959-992.

Sibiano, P., Agasisti, T. (2013). Efficiency and heterogeneity of public spending in education among Italian Regions. Journal of Public Affairs, 13(1), 12-22. 
Silva, J. L. M., Almeida, J. C. L. (2012). Eficiência no gasto público com educação: uma análise dos municípios do Rio Grande do Norte. Planejamento e Políticas Públicas, (39).

Souza, C. V. N. D., Jr., Gasparini, C. E. (2006). Análise da equidade e da eficiência dos estados no contexto do federalismo fiscal brasileiro. Estudos Econômicos (São Paulo), 36(4), 803-832.

Sueyoshi, T., Goto, M., Omi, Y. (2010). Corporate governance and firm performance: Evidence from Japanese manufacturing industries after the lost decade. European Journal of Operational Research, 203(3), 724-736.

Tschaffon, P. B., Angulo Meza, L. (2014). Assessing the efficiency of the electric energy distribution using Data Envelopment Analysis with undesirable outputs. Latin America Transactions, IEEE (Revista IEEE America Latina), 12(6), 1027-1035.

Whiteley, P. F. (2000). Economic growth and social capital. Political Studies, 48(3), 443-466.

Wilbert, M. D., D’Abreu, E. C. C. F. (2013). Eficiência dos gastos públicos na educação: análise dos municípios do estado de alagoas. Advances in Scientific and Applied Accounting, 6(3), 348-372.

Will, A. R. (2014). Eficiência dos Estados Brasileiros nos Gastos com Educação: Um Estudo Comparativo de Recursos Utilizados e Resultados Alcançados. (Dissertação de mestrado, Universidade Federal de Santa Catarina, Florianópolis, SC, Brasil).

Zhu, J. (2000). Multi-factor performance measure model with an application to Fortune 500 companies. European journal of operational research, 123(1), 105-124

Zoghbi, A. C. P., Matos, E. H. C. D., Rocha, F. F., Arvate, P. R. (2009). Mensurando o desempenho e a eficiência dos gastos estaduais em educação fundamental e média. Estudos Econômicos (São Paulo), 39(4), 785-809.

\section{BY-NC-ND}

